Francophonies d'Amérique

TRANCOPHONIES

DAMERRE

\title{
Le Triangle canadien-français au tournant des années 1960. Le Conseil de la vie française en Amérique, la Société Saint-Jean-Baptiste de Montréal et l'Ordre de Jacques-Cartier
}

\section{Gratien Allaire}

Numéro 17, printemps 2004

URI : https://id.erudit.org/iderudit/1005284ar

DOI : https://doi.org/10.7202/1005284ar

Aller au sommaire du numéro

Éditeur(s)

Les Presses de l'Université d'Ottawa

ISSN

1183-2487 (imprimé)

1710-1158 (numérique)

Découvrir la revue

Citer cet article

Allaire, G. (2004). Le Triangle canadien-français au tournant des années 1960. Le Conseil de la vie française en Amérique, la Société Saint-Jean-Baptiste de Montréal et l'Ordre de Jacques-Cartier. Francophonies d'Amérique, (17),

107-116. https://doi.org/10.7202/1005284ar d'utilisation que vous pouvez consulter en ligne. 


\title{
LE TRIANGLE CANADIEN-FRANÇAIS AU TOURNANT DES ANNÉES 1960. LE CONSEIL DE LA VIE FRANÇAISE EN AMÉRIQUE, LA SOCIÉTÉ SAINT-JEAN-BAPTISTE DE MONTRÉAL ET L'ORDRE DE JACQUES-CARTIER*
}

\author{
Gratien Allaire \\ Université Laurentienne
}

L

dienne acceptent la thèse de la fin du Canada français, ou tout au moins de son éclatement, au cours des années 1960. J'ai pour ma part avancé l'hypothèse d'une métamorphose, qui suppose plutôt une modification profonde du Canada français en francophonie canadienne, mais il n'en reste pas moins que le Canada français connaît des changements en profondeur au cours de la période de la Révolution tranquille québécoise. Yves Frenette (1998), Gaétan Gervais ${ }^{1}$ et Marcel Martel (1997), entre autres $^{2}$, centrent leur interprétation sur les États généraux du Canada français qui ont eu lieu en 1967 et en 1969. Selon eux, cette grande rencontre des Canadiens français met fin au Canada français tel qu'on l'a connu tout au long de la première moitié du vingtième siècle. Elle a été l'expression du nationalisme canadien-français devenu québécois, donc centré sur le Québec, et a marqué le rejet par ce dernier du reste du Canada français, l'exclusion des minorités de langue française, considérées comme perdues. Il y a bien quelques voix discordantes, comme celle de la sociologue Linda Cardinal (1998, p. 213-232), mais la thèse de la rupture demeure largement acceptée.

Cette thèse de la centralité des États généraux est assise principalement sur l'évolution du discours clérico-nationaliste canadien-français, plus particulièrement celui du Québec. En fait, elle considère que le mouvement nationaliste constitue tout le Québec, et ce n'est pas le cas. Elle oublie que le gouvernement québécois a versé d'importantes subventions aux groupes minoritaires de façon régulière au cours des années 1960 par l'intermédiaire de son Service du Canada français d'outre frontières ${ }^{3}$. Cette interprétation met aussi de côté ou minimise certains éléments constitutifs du Canada français, dont le rôle du clergé et des institutions que ce dernier possède ou contrôle. Ses défenseurs traitent des trois grandes associations qui ont soutenu le Canada français, mais ils ne les voient pas ensemble et ne tiennent pas compte de la dynamique qui les anime ou des rivalités qui les opposent. Pourtant, sociétés Saint-Jean-Baptiste, Ordre de Jacques-Cartier et Conseil de la vie française en Amérique ont, pendant les années 1940 et 1950, agi ensemble pour la survivance du Canada français et des minorités. Martel fait exception, mais il subordonne tout le mouvement à l'Ordre de Jacques-Cartier ${ }^{4}$.

\footnotetext{
* Texte d'une communication présentée à Paris, le 19 mai 2001, lors du colloque «Francophonie au pluriel» organisé par L'Année francophone internationale (AFI). Ce texte, publié dans Francophonies d'Amérique avec la permission de l'AFI, est également disponible sur le site de l'organisme (http://www.ulaval.ca/afi/colloques/colloque2001/ actes/textes/allaire.htm).
} 
Puisque ces trois associations ont joué un rôle clé dans le maintien du Canada français, il est important de voir leur rôle au cours des années 1960. Ces années de remise en question sont marquées par une lutte à finir entre Montréal et Ottawa, lutte qui s'est faite à l'intérieur de l'Ordre de Jacques-Cartier, mais qui oppose en réalité les jeunes qui se retrouvent dans la Société Saint-Jean-Baptiste de Montréal aux anciens de l'Ordre de Jacques-Cartier. Dans cette bataille, le Conseil de la vie française, qui avait son siège à Québec et qui avait coordonné les campagnes des années 1940 et 1950, n'a joué aucun rôle. C'est que le Conseil ne s'est pas renouvelé et qu'il est alors composé de gérontes qui se sont laissé dépasser par les événements. C'est pourquoi il n'intervient pas; c'est ce qui explique aussi qu'il ne remplit pas le vide laissé par la disparition de l'Ordre de Jacques-Cartier en 1965. Finalement, les nationalistes canadiensfrançais à tendance québécoise et la Société Saint-Jean-Baptiste de Montréal sont restés tout seuls sur le terrain et ils ont pris le contrôle des États généraux.

Nous commencerons par un bref tour d'horizon de chacune des trois associations, pour constater le peu de publications sur le sujet. Nous passerons ensuite en revue le débat - la guerre idéologique, devrait-on dire - qui oppose Montréal à Ottawa, la conception montréalaise à la conception outaouaise. Nous nous attarderons finalement sur la composition du Conseil de la vie française au début des années 1960, composition qui explique en bonne partie le peu d'importance du Conseil dans le débat en cours. Le CVFA s'est sclérosé et il est dépassé par la discussion et par les événements. On comprendra qu'il s'agit ici d'un travail exploratoire plutôt que d'une étude complète; seul un aspect de ces grands changements est abordé et les conclusions ne sont que préliminaires.

\section{Les sociétés Saint-Jean-Baptiste}

Il n'existe pas de monographie publiée sur l'ensemble des sociétés Saint-Jean-Baptiste. L'ouvrage le plus important porte sur la Société Saint-Jean-Baptiste de Montréal (SSJBM) ; c'est celui de l'historien clérico-nationaliste Robert Rumilly. Il s'agit d'une chronique, à la Rumilly, qui couvre l'évolution de la société jusqu'en 1948, et repose sur les procès-verbaux de ses réunions. La description est centrée sur Montréal et le Québec uniquement. Par exemple, elle ne fait aucune mention des voyages de la survivance, qui ont, entre 1925 et 1930, amené à Montréal plusieurs centaines de Canadiens français des Prairies, même si la SSJBM a contribué à leur réception 5 (1971, tome XXVIII, p. 310-312; tome XXIX, p. 219).

Pourtant, durant toute son existence, la Société Saint-Jean-Baptiste a été au cœur de l'organisation du Canada français et de l'évolution de son idéologie. Fondée en 1834 à Montréal par Ludger Duvernay, elle a pour mission de défendre les droits des Canadiens français. Son rôle dépasse toutefois l'action politique. Elle sert de point de rassemblement ; elle organise des célébrations, dont la fête de la Saint-Jean-Baptiste, le 24 juin de chaque année. C'est une des premières associations que les Canadiens français établissent pour se regrouper dans les zones de nouvel établissement. Elles se répandent rapidement en Ontario et en Nouvelle-Angleterre. Les sociétés locales se forment très tôt au Manitoba et elles sont suffisamment nombreuses en 1908 pour que l'archevêque de Saint-Boniface, Mgr Adélard Langevin, les invite à former une fédération provinciale (Lapointe et Tessier, 1986, p. 208). Elle se trouve à Battleford en Saskatchewan avant 1885 et, en 1909, on vote la formation d'une Société Saint-Jean-Baptiste de la Saskatchewan (p. 208). Elle est établie à Edmonton en Alberta dès 1894 (Hart, 1981, p. 32). Lors de la crise du Règlement XVII en Ontario, elle offre son appui, finan- 
cier et moral à la population de langue française de l'Ontario (Dufresne et al., 1988, p. 347). Il n'y a qu'en Acadie qu'on ne trouve pas la SSJB. La population acadienne s'est donnée des symboles propres et des organismes bien à elle dès les années 1880 , lors des grandes conventions nationales (Allaire, 1999, p. 47).

Durant toute la première moitié du vingtième siècle, la société Saint-Jean-Baptiste a été de toutes les disputes pour la défense du Canada français, et elle a appuyé fortement tous les mouvements qui favorisaient la " survivance » dans les autres provinces canadiennes comme au Québec. Qu'il s'agisse de la Fédération des sociétés Saint-JeanBaptiste ou des sociétés locales, y compris celle de Montréal, leur appui est assuré, même s'il arrive parfois des discussions et des divergences de vue, comme entre l'Ordre et la SSJBM au sujet de la conscription et de la participation à la Deuxième Guerre mondiale (Martel, 1997, p. 44-46).

\section{L'Ordre de Jacques-Cartier}

L'Ordre de Jacques-Cartier, autrement appelé « la Patente », a fait l'objet d'un certain nombre d'analyses et d'études, la plus récente ayant été présentée par le Réseau de l'information le 8 mai 2001. L'émission utilise les archives de l'Ordre, dont les Archives nationales du Canada viennent d'ouvrir l'accès, selon les directives des syndics de l'organisme; le Centre de recherche en civilisation canadienne-française de l'Université d'Ottawa a fait de même. L'Ordre de Jacques-Cartier, un mystère dévoilé, du réalisateur Robert Verge (2001), retrace en une heure l'historique de l'Ordre et insiste sur son caractère secret. Verge montre l'Ordre comme l'organisme qui a noyauté le Canada français et l'a mené des années 1930 jusqu'à sa dissolution en 1965. Il en présente les membres comme faisant preuve $\mathrm{d}^{\prime}$ " une docilité que l'on s'explique à peine de nos jours ». Il ajoute qu' " [i]l faut savoir que toutes les élites canadiennes-françaises ont déjà été télécommandées par un groupe occulte et clandestin cloîtré à Ottawa. ». La principale limite de l'émission, c'est de présenter l'Ordre comme le seul organisme du Canada français durant toute cette période.

Si l'on met de côté le livre de Roger Cyr (1964), qui relève davantage de la dénon-

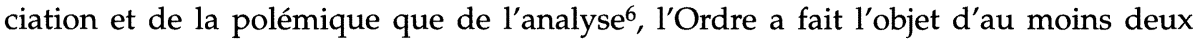
autres travaux : l'ouvrage de G.-Raymond Laliberté, en 1983, et un article plus récent de Gabriel Bertrand (1998).

L'ancien syndicaliste devenu politologue et sociologue G.-Raymond Laliberté présente une analyse magistrale de l'Ordre (1983). Elle est basée sur des entrevues avec des personnalités de l'Ordre, comme J.-Z.-Léon Patenaude, et sur des sources documentaires accessibles alors seulement avec la permission des syndics de l'Ordre, comme le fonds de l'Ordre déposé au Centre de recherche en civilisation canadiennefrançaise de l'Université d'Ottawa. Laliberté a également consulté le fonds RosaireMorin, déposé à l'Institut d'histoire de l'Amérique française, devenu depuis le Centre de recherche Lionel-Groulx. Après avoir analysé le fonctionnement de l'Ordre et ses campagnes, Laliberté conclut à « l'utopie d'un césarisme laurentien » (p. 357-364). Une autre de ses conclusions - la suite à voir entre l'Ordre et le Parti québécois - avait fait la chronique des journaux lors de la parution en 1983.

Contrairement à Laliberté, qui voit l'Ordre dans le contexte de l'évolution du Québec principalement, le sociologue Gabriel Bertrand, dans une communication présentée en 1995 lors du colloque annuel du Réseau de la recherche sur la francophonie canadienne, place l'Ordre dans le cadre canadien-français et étudie plus particulièrement son rôle auprès des minorités. Se basant aussi sur le fonds Rosaire-Morin et y ajoutant le fonds Théo-Godin du Centre d'études acadiennes de l'Université de Monc- 
ton, Bertrand énumère les principales activités de l'Ordre en faveur des minorités et décrit la controverse des dernières années de l'organisme. Il insiste sur le conflit entre deux tendances à l'intérieur de l'Ordre, la tendance canadienne-française prônée par la chancellerie à Ottawa et la tendance québécoise, de plus en plus forte, préconisée par le groupe de Montréal. Il y avait, selon Bertrand, « une opposition marquée entre l'aile québécoise, dans laquelle les Montréalais jouaient un rôle primordial, et la chancellerie » $(1995,1996$, p. 40). Rosaire Morin, que l'on retrouve au comité organisateur des États généraux (Martel, 1998, p. 39), est l'un des meneurs du mouvement réformiste montréalais.

L'Ordre de Jacques-Cartier est fondé à Ottawa en 1926 par un groupe de Canadiens français, dont plusieurs fonctionnaires fédéraux. C'est une société secrète, à l'image de l'Ordre d'Orange, des francs-maçons, sociétés protestantes de langue anglaise, et des Chevaliers de Colomb, société catholique irlandaise. Il vise la promotion des Canadiens français dans la fonction publique fédérale et compte « former un rempart solide contre l'assimilation des minorités canadiennes-françaises " (Bertrand, 1995, 1996, p. 15 ; Laliberté, 1983, p. 42-48). L'Ordre choisit ses membres par cooptation, et ne deviennent membres que ceux qu'il a choisis. Tout comme les sociétés Saint-Jean-Baptiste, l'Ordre a rapidement pris de l'expansion à l'extérieur d'Ottawa et de l'Ontario. Dès 1927, il compte sept commanderies, dont trois au Québec. On le retrouve dans l'Ouest en 1930 et dans les Maritimes en 1932. En 1934, moins de dix ans après sa fondation, il a formé au total 59 commanderies, dont 39 au Québec et 13 en Ontario (Laliberté, p. 44). L'Ordre répondait donc à un besoin. L'organisme comptait 9822 membres en 1953 (Bertrand, p. 18), 11192 au printemps 1960 (Laliberté, p. 96). En 1964, l'année précédant sa dissolution, ses effectifs avaient fondus, mais l'Ordre regroupait encore 5076 membres, répartis en 466 commanderies (Bertrand, p. 18).

L'Ordre possède une structure très hiérarchisée et fonctionne par mot d'ordre. Il s'attend au respect de ces directives et à la plus grande discrétion de ses membres. Il a pour stratégie de noyauter les organismes et les institutions, pour les amener à agir dans le sens proposé et pour atteindre les objectifs fixés de survivance canadiennefrançaise. L'Ordre a parrainé plusieurs campagnes canadiennes-françaises, pour le bilinguisme, pour le financement des communautés. Selon Laliberté, et selon Verge, il est derrière toutes les initiatives pour le maintien et la promotion du Canada français, d'un bout à l'autre du pays.

La scission des années 1960 qui mène à la dissolution de l'Ordre a été largement analysée par Laliberté et par Bertrand. Les deux auteurs y ont vu d'abord et avant tout la lutte du nationalisme québécois en plein essor et du nationalisme canadien-français de plus en plus marginalisé. Cette thèse de l'opposition des idéologies se heurte au fait que Rosaire Morin et le groupe montréalais ont voulu réformer l'Ordre de l'intérieur, pour lui donner une couleur plus québécoise et plus représentative de la majorité des membres. La fondation subséquente de l'Ordre de Jean-Talon peut être vue comme une volonté de maintenir l'Ordre, mais on peut aussi y voir une preuve que le groupe de Morin voulait confisquer l'Ordre au bénéfice de la cause nationaliste québécoise. Il faut aussi placer dans la balance le fait que l'Ordre était en perte de vitesse depuis le début des années 1960.

On peut se demander à quel point l'Ordre a été réceptif à la réforme, au renouvellement et à la discussion des nouvelles réalités canadiennes-françaises des années 1960. Il faut constater que, devant cette volonté de réforme, la chancellerie s'en est tenue à la position traditionnelle et lui a opposé, à toutes fins pratiques, une fin de non-recevoir. Le lien Église catholique - communauté demeure très fort. L'Ordre lui-même ne se 
renouvelle pas, les chanceliers demeurant les grands défenseurs de l'idéologie clériconationaliste. «La force de l'Ordre de Jacques-Cartier, écrit l'historien Martel, réside dans son caractère secret garanti par une sélection minutieuse de ses membres. Il faut être sélectionné par l'Ordre pour en devenir membre, ce qui permet de contrôler la croissance des cellules locales au Canada » (Martel, 1997, p. 34). C'est ce qui permet également de contrôler l'évolution des idées et de maintenir le statu quo.

\section{Le Conseil de la vie française}

Le Conseil de la vie française en Amérique a été étudié en profondeur par Marcel Martel, dont l'ouvrage porte sur les rapports entre le Québec et la francophonie canadienne entre 1867 et 1975 . Le titre du livre est assez indicateur de l'interprétation avancée par Martel: Le deuil d'un pays imaginé. L'historien y traite de la rupture, de l'éclatement du Canada français, après une analyse qui fait beaucoup de place au Conseil de la vie française en Amérique dans l'évolution de la francophonie dans les années 1940 et 1950. Puis, il lui accorde une place centrale au débat idéologique menant aux États généraux au cours des années 1960.

Le Conseil a été fondé en 1937 à la suite du Deuxième Congrès de la langue française. Il porte pendant un certain temps le nom du Comité permanent de la Survivance française. Il se donne comme objectifs, selon sa charte de 1940, « le soutien et la défense des intérêts nationaux des populations de langue française et le maintien des traditions de l'esprit français, en Amérique du Nord » (Le Comité permanent de la Survivance française en Amérique, 1940, p. 13, cité dans Martel, 1997, p. 43-44). Le Comité/Conseil a son siège à Québec, à l'Université Laval, où se trouve son secrétaire permanent, sinon éternel, Mgr Paul-Émile Gosselin.

Le Conseil recrute des membres de toutes les régions de l'Amérique française et il voit son action comme portant sur son ensemble, Québec comme Acadie, Ontario et Ouest, Canada comme Nouvelle-Angleterre et Louisiane. Ses campagnes sont politiques ; elles sont surtout d'appui aux minorités, appui financier comme moral. Le Conseil coordonne l'action des divers acteurs, incluant les sociétés Saint-Jean-Baptiste. Ses plus grandes réussites sont sans doute la radio française dans l'Ouest (Martel, p. 51-56) et la campagne de financement pour la fondation du quotidien L'Évangéline en 1942 (p. 47-51).

Au cours des années 1960, le Conseil n'est plus en mesure de jouer ce rôle de coordination et de rassemblement. On peut le considérer comme un spectateur impuissant dans le débat qui oppose Montréal et Ottawa. On trouve dans sa composition un facteur d'explication de cette position. Le tableau 1 montre à quel point le Conseil a omis de se renouveler.

Selon la liste dressée par Marcel Martel, le Conseil compte 40 membres en 1960. De ce nombre, la moitié sont du Québec, soit neuf de la région de Montréal et neuf de la région de Québec; les deux autres sont de Sherbrooke et de Chicoutimi. L'Ontario occupe cinq sièges, l'Acadie cinq aussi (deux pour le Nouveau-Brunswick, deux pour la Nouvelle-Écosse et un pour l'Île-du-Prince-Édouard). Les quatre provinces de l'Ouest ont un siège chacune et la Nouvelle-Angleterre, cinq. Cette représentation est réduite à 36 en 1967, les régions de Québec et de Montréal, comme d'ailleurs la Nouvelle-Angleterre et l'Ontario ayant un représentant de moins chacun. L'équilibre Québec - extérieur du Québec demeure. La représentation s'est modifiée en 1958, par la fin de la représentation des recteurs des trois universités (Laval, Montréal et Ottawa). Le changement des membres fait que certaines régions ne sont plus représentées : Drummondville, Trois-Rivières, la Louisiane. 
Le Conseil de 1960 a du galon. Vingt-deux membres, plus de la moitié du Conseil, sont entrés en fonction avant 1951 et siègent donc depuis au moins une dizaine d'années. Sept représentent ce que l'on pourrait appeler la vieille garde : ils sont membres depuis les tout débuts de l'organisme et sont d'un âge respectable. Paul-Émile Gosselin, prêtre et âme du Conseil depuis le début, est né en 1909 ; il est entré au Conseil en 1937 et dirige le secrétariat depuis 19387. Henri Boisvert, notaire de Québec, en fait partie depuis $1937^{\circ}$, de même que Joseph-Henri Blanchard, professeur de l'Île-duPrince-Édouard né en 1881 - il a 79 ans en $1960^{\circ}$. Parmi ceux qui sont entrés en 1938, siègent encore en 1960 Arthur Joyal, prêtre de l'Ontario $^{10}$, né en 1883, J.A. Symaune Plouffe, juge de $1^{\prime}$ Ontario, né en $1893^{11}$, et Adrien Verrette, prêtre de la NouvelleAngleterre, né en $1897^{12}$. Enfin, Adrien Pouliot, professeur de mathématiques et doyen de la faculté des Sciences de l'Université Laval, y est depuis 193913. Parmi ces gérontes, quatre sont de l'extérieur du Québec. On peut percevoir un début de renouvellement : 12 nouveaux membres sont nommés entre 1957 et 1959, dont trois pour la NouvelleAngleterre et quatre pour le Canada hors Québec. Ce renouvellement a toutefois ses limites, puisque le Conseil contrôle les nominations (Martel, 1997, p. 57).

Le Conseil de 1967 est différent, mais il compte encore des membres de longue date. Treize des 36 membres, plus du tiers du Conseil, ont été nommés avant 1951, dont trois avant 1939 (Gosselin, Verrette et Pouliot). Seulement huit membres sont entrés au cours des années 1950. Il est difficile de parler de véritable renouvellement puisque moins de la moitié du Conseil, 16 membres, ont été nommés depuis 1960, dont huit pour le Canada hors Québec et un pour la Nouvelle-Angleterre. En fait, le renouvellement est plus prononcé pour le Canada hors Québec que pour le Québec même : huit des 13 membres changent entre 1960 et 1967. Si l'on examine la représentation du Québec, l'image est la suivante : huit des 19 membres en provenance du Québec ont été nommés avant 1951, y compris les éternels Gosselin et Pouliot, six des huit membres de la région de Montréal siégeaient déjà avant 1960 et il en est de même pour la région de Québec (six sur huit).

Si l'on examine de près l'évolution de la composition du Conseil de la vie française durant ces années d'effervescence et de discussion, il reste pareil à lui-même et s'en assure par la nomination des nouveaux membres, mais le nombre de ces derniers est limité. Formé au cours des années du clérico-nationalisme triomphant, le Conseil continue cette idéologie, par sa représentation en provenance du Québec et par le choix du renouvellement de ceux de l'extérieur. Dans ces conditions, il y a peu de chance que le Conseil puisse contribuer véritablement au débat mené vigoureusement par le groupe de Montréal, autour de la Société Saint-Jean-Baptiste de Montréal. Il y a également peu de chance que les " réformistes " soient intéressés à faire partie du Conseil. Ce dernier est de plus en plus dépassé. L'Ordre de Jacques-Cartier disparu, le Conseil de la vie française en Amérique déconnecté, le terrain reste à la Société Saint-Jean-Baptiste de Montréal. Et il n'y a pas de véritable débat sur l'orientation du nationalisme canadien-français lors des États généraux. On peut dire que les jeux sont faits et que le Groupe de Montréal n'a plus d'opposition.

\section{Conclusion}

L'évolution du mouvement nationaliste au début des années 1960 et sa résultante lors des États généraux se comprennent mieux lorsque l'on suit, ensemble, les trois grandes associations canadiennes-françaises. Il ressort de ce tour d'horizon et de cette analyse de la composition du Conseil de la vie française en Amérique au cours des 
années 1960 que les structures et les modes d'action de l'Ordre de Jacques-Cartier et du Conseil de la vie française en Amérique, qui les ont bien servis des années 1930 aux années 1960, les ont empêchés de se renouveler dans les années 1960. Face au groupe dynamique de Montréal, l'Ordre résiste et continue d'opposer la volonté hiérarchique et l'inflexibilité des mots d'ordre. Le Conseil de la vie française aurait pu continuer de jouer le rôle de rassembleur qu'il a tenu au cours des années 1950 en particulier, mais son manque de renouvellement le place davantage du côté de l'Ordre de Jacques-Cartier et du maintien de l'ordre établi et de l'idéologie clérico-nationaliste. Il n'influence plus la discussion.

Il faut pousser l'analyse un peu plus loin. Au-delà de la lutte nationaliste, à l'intérieur de l'Ordre de Jacques-Cartier comme à l'intérieur de tout le mouvement nationaliste canadien-français, il y a une lutte de générations. Les tenants de l'ordre établi au sein de l'Ordre de Jacques-Cartier ont été battus par les réformistes du groupe de Montréal. Les gérontes du Conseil de la vie française n'ont pas pu intervenir. L'organisme qui ressort comme le grand gagnant des discussions des années 1960, ce sont les sociétés Saint-Jean-Baptiste. L'Ordre de Jacques-Cartier disparaît en 1965 et ses remplacements sont de très courte durée. Le Conseil de la vie française perd de plus en plus de son importance. C'est la Fédération québécoise des sociétés Saint-Jean-Baptiste du Québec qui convoque les États généraux (Morin, cité dans Martel, 1997, p. 39) et Rosaire Morin est l'un de ses trois organisateurs principaux. 


\section{Tableau 1}

Conseil de la vie française en Amérique Répartition des membres selon la date d'entrée en fonction et la provenance

(Québec - Q, hors Québec - HQ et Nouvelle-Angleterre - NA ; 1960 et 1967)

\begin{tabular}{|c|c|c|c|c|c|c|c|c|}
\hline \multirow[b]{2}{*}{$\begin{array}{l}\text { Entrée en } \\
\text { fonction }\end{array}$} & \multicolumn{4}{|c|}{ Membres du Conseil en 1960} & \multicolumn{4}{|c|}{ Membres du Conseil en 1967} \\
\hline & Q & HQ & NA & Total & $Q$ & $\mathrm{HQ}$ & NA & Total \\
\hline $1937-1939$ & 3 & 3 & 1 & 7 & 2 & & 1 & 3 \\
\hline 1943-1946 & 2 & 5 & 1 & 8 & - & 3 & 1 & 4 \\
\hline $1949-1951$ & 6 & 1 & - & 7 & 6 & - & - & 6 \\
\hline 1953 & 1 & & & 1 & 1 & & & 1 \\
\hline 1954 & 2 & 1 & & 3 & 1 & & & 1 \\
\hline 1955 & 1 & & & 1 & 1 & & & 1 \\
\hline 1956 & & 1 & & 1 & & & & \\
\hline 1957 & & 1 & 2 & 3 & & & & \\
\hline 1958 & & 3 & 1 & 4 & & 2 & 1 & 3 \\
\hline 1959 & 5 & & & 5 & 2 & & & 2 \\
\hline \multicolumn{9}{|l|}{1960} \\
\hline 1961 & & & & & 1 & & & 1 \\
\hline 1962 & & & & & 1 & 1 & & 2 \\
\hline 1963 & & & & & & 3 & & 3 \\
\hline 1964 & & & & & & 1 & 1 & 2 \\
\hline 1965 & & & & & 2 & & & 2 \\
\hline 1966 & & & & & 3 & 3 & & 6 \\
\hline Total & 20 & 15 & 5 & 40 & 19 & 13 & 4 & 36 \\
\hline
\end{tabular}

Source : Compilé à partir de la liste des membres du Conseil fournie par Marcel Martel dans Le deuil d'un pays imaginé ; rêves, luttes et déroute du Canada français : les rapports entre le Québec et la francophonie canadienne (18671975), Ottawa, Presses de l'Université d'Ottawa, 1997, p. 179-183. 


\section{Le triangle canadien-français au tournant des années 1960}

\section{NOTES}

1. Gaétan Gervais a une opinion plus nuancée : "Les États généraux n'ont ni causé, ni précipité, la fin du Canada français. Ils en apportèrent seulement une preuve éclatante. Avec les États généraux s'éteignait un monde, celui du Canada français ". (Voir Martel, 1998, p. 132).

2. Dans un ouvrage qui vient tout juste de paraître, Michel Bock analyse aussi cette notion pour le Nord de l'Ontario.

3. D'une certaine manière, il s'agit là du déplacement vers l'État d'un autre des rôles sociaux de l'Église catholique et du mouvement d'action sociale. (Voir Allaire, 1993, p. 229-245 ; Savas, 1988, p. 11-54 ; Cadrin, 1991, p. 269-281).

4. Selon Martel, «les associations provinciales sont ses exécutantes dans les milieux francophones minoritaires » et «la Fédération des Sociétés Saint-Jean-Baptiste du Québec (SSJBQ) ou le Conseil [de la vie française en Amérique] lui servent de couverture ». Martel, 1997, p. 35.

5. Pourtant Rumilly en fait mention dans Histoire de la province de Québec.

6. L'ouvrage fait partie de la contestation qui a mené à la dissolution de l'Ordre.

7. Gosselin est prêtre depuis 1934. Professeur de philosophie au Petit Séminaire de Québec, il enseigne ensuite la philosophie de la nature à l'Université Laval de 1944 à 1955. Il est aussi directeur de L'Action catholique de 1953 à 1962, de la Société du parler français, de la revue Vie française, il organise les voyages de la Liaison française (Dufresne et al., 1988, p. 168).

8. Le notaire Boisvert est trésorier du Conseil de 1942 à 1955. Il est également membre de la Société Saint-JeanBaptiste de Québec et de la Société canadienne d'établissement rural (Martel, 1997, p. 59).

9. Blanchard, né en 1881, enseigne le français et l'histoire, puis est vice-principal du collège Prince of Wales de Charlottetown, puis à l'Université Mount Allison. Il a été président de la Société Saint-Thomas d'Aquin et de la Société nationale des Acadiens. Ses publications portent sur les Acadiens de l'île-du-Prince-Édouard. (Dufresne et al., 1988, p. 53).

10. Arthur Joyal, prêtre oblat est né au Manitoba en 1883. Après son scolasticat à Ottawa, il sert au Cap-de-laMadeleine, puis à Montréal et ensuite en Ontario. Il dirige le secrétariat de l'Association canadienne-française d'éducation de l'Ontario. Il est toutefois jugé timide dans ses revendications ; c'est sa façon de faire accepter la communauté franco-ontarienne (Dufresne et al., 1988, p. 188).

11. Né en 1893, Plouffe a fait son droit à Osgoode Hall. Il pratique à Sudbury de 1919 à 1936 et devient ensuite juge à North Bay. Il est l'un des fondateurs de l'ACFEO et préside aussi la Société historique du Nouvel Ontario. Actif au sein de l'Ordre de Jacques-Cartier, Plouffe est un « défenseur convaincu des droits des FrancoOntariens » (Dufresne et al., 1988, p. 286).

12. Verrette, né en 1897, est prêtre depuis 1921. Vicaire, puis curé dans les paroisses du New Hampshire, il a écrit La croisade franco-américaine en 1938 ; il est éditeur de La Vie franco-américaine de 1937 à 1952 et du Bulletin de la société historique franco-américaine (Dufresne et al., 1988, p. 378).

13. Pouliot est doyen de 1940 à 1956. Il est aussi gouverneur de la Société Radio-Canada à partir de 1939, viceprésident du bureau des gouverneurs en 1956 et brièvement président en 1958. Il entre au Conseil comme représentant de la Société du parler français (Martel, 1997, p. 59).

\section{BIBLIOG RAPHIE}

AlLAIRE, Gratien (1993), « De l'Église à l'État : le financement des organismes francophones de l'Ouest, 19561970 ", dans Jean LAFONTANT et al. (dir.), L'État et les minorités, textes du colloque tenu au Collège de Saint-Boniface les 6 et 7 novembre 1992, Saint-Boniface, Les Éditions du Blé et Presses universitaires de Saint-Boniface, p. 229-245.

AlLAIRE, Gratien (1999), La francophonie canadienne, Québec, CIDEF-AFI ; Sudbury, Prise de Parole.

BERTRAND, Gabriel (1995/1996), "L'Ordre de Jacques-Cartier et les minorités francophones ", dans Gratien AllAIRE et Anne GILBERT (dir.), Francophonies plurielles : communications choisies, Colloques du Regroupement pour la recherche sur la francophonie canadienne organisés dans le cadre du congrès annuel de l'ACFAS, Chicoutimi, 1995 et Montréal, 1996, Sudbury, Institut franco-ontarien, p. 13-67. 
Bock, Michel (2001), Comment un peuple oublie son nom : la crise identitaire franco-ontarienne et la presse française de Sudbury (1960-1975), Sudbury, Institut franco-ontarien, « Ancrages ».

CADRIN, Gilles (1992), "L'affirmation des minorités francophones depuis la Révolution tranquille ", dans Gratien AllaIRe, Paul DubÉ et Gamila Morcos (dir.), Après dix ans- bilan et prospective : les actes du onzième Colloque du Centre d'études franco-canadiennes de l'Ouest tenu à la Faculté Saint-Jean, Université de l'Alberta, du 17 au 19 octobre 1991, Edmonton, Institut de recherche de la Faculté Saint-Jean, p. 269-281.

CARDINAL, Linda (1998), "Le Canada français à la lumière des États généraux: critique de la thèse de la rupture ", dans Marcel MARTEL, avec la collaboration de Robert CHOQUETTE, Les États généraux du Canada français, trente ans après: actes du colloque tenu à l'Université d'Ottawa les 5, 6 et 7 novembre 1997, Ottawa, Centre de recherche en civilisation canadienne-française de l'Université d'Ottawa, p. 213-232.

CYR, Roger (1964), La Patente, Montréal, Les Éditions du Jour.

DUFRESNE, Charles et al. (1988), Dictionnaire de l'Amérique française : francophonie nord-américaine hors-Québec, Ottawa, Presses de l'Université d'Ottawa.

FRENETTE, Yves avec la collaboration de Martin PÂQUET (1998), Brève histoire des Canadiens français, Montréal, Boréal.

GERVAIS, Gaétan (1998), "La présence de l'Ontario aux États généraux du Canada français (1966-1969) », dans Marcel MARTEL, avec la collaboration de Robert CHOQUETTE, Les États généraux du Canada français, trente ans après : actes du colloque tenu à l'Université d'Ottawa les 5, 6 et 7 novembre 1997, Ottawa, Centre de recherche en civilisation canadienne-française de 1'Université d'Ottawa, p. 117-132.

HART, E. J. (1981), Ambitions et réalités : la communauté francophone d'Edmonton, 1795-1935, Edmonton, le Salon d'histoire de la francophonie albertaine, p. 32.

LALIBERTÉ, G.-Raymond (1983), Une société secrète: l'Ordre de Jacques-Cartier, Montréal, Hurtubise HMH, "L'homme dans la société ».

LAPOINTE, Richard et Lucille TESSIER (1986), Histoire des Franco-Canadiens de la Saskatchewan, Regina, Société historique de la Saskatchewan.

L'Ordre de Jacques-Cartier, un mystère dévoilé (2001), scénario et réalisation de Robert VERGE, production de Pierre L. TOUCHETTE pour Amérimage-Spectra en collaboration avec RDI, documentaire d'une heure.

MARTEL, Marcel (1997), Le deuil d'un pays imaginé ; rêves, luttes et déroute du Canada français : les rapports entre le Québec et la francophonie canadienne (1867-1975), Ottawa, Les Presses de l'Université d'Ottawa.

MARTEL, Marcel, avec la collaboration de Robert CHOQUETTE (1998), Les États généraux du Canada français, trente ans après : actes du colloque tenu à l'Université d'Ottawa les 5, 6 et 7 novembre 1997, Ottawa, Centre de recherche en civilisation canadienne-française de l'Université d'Ottawa.

MORIN, Rosaire (1998), «Les États généraux du Canada français 1967-1969 », dans Marcel MARTEL, avec la collaboration de Robert CHOQUETTE, Les États généraux du Canada français, trente ans après : actes du colloque tenu à l'Université d'Ottawa les 5, 6 et 7 novembre 1997, Ottawa, Centre de recherche en civilisation canadienne-française de l'Université d'Ottawa, p. 39-46.

RuMILly, Robert (1971), Histoire de la province de Québec, tome XXVIII, La rue Saint-Jacques, Montréal et Paris, Fides.

RUMILly, Robert (1971), Histoire de la province de Québec, tome XXIX, Vers l'âge d'or, Montréal et Paris, Fides.

RuMILly, Robert (1975), Histoire de la Société Saint-Jean-Baptiste de Montréal : des Patriotes au Fleurdelisé, 1834-1948, Montréal, L'Aurore.

SAVAS, Daniel (1988), «L'impact des politiques d'aide du Secrétariat d'État sur l'évolution financière de la Fédération des Franco-Colombiens ", dans Monique BOURNOT-TRITES, William BRUNEAU et Robert RoY (dir.), Les outils de la francophonie : actes du sixième Colloque du Centre d'études franco-canadiennes de l'Ouest, tenu à Richmond, Colombie-Britannique, les 10 et 11 octobre 1986, Saint-Boniface, Man., CEFCO ; Vancouver, University of British Columbia, p. 11-54. 